\title{
Performances of Mini Parasep SF stool concentrator kit , Kato-Katz and formalin-ethyl acetate concentration methods for diagnosis of opisthorchiasis among residents in an endemic community in northeast Thailand
}

Kulthida Y. Kopolrat

Kasetsart University

Seri Singthong

Ministry of Public Health Thailand

Narong Khuntikeo

Khon Kaen University

Watcharin Loilome

Khon Kaen University

Chanika Worasith

Khon Kaen University

Chutima Homwong

Khon Kaen University

Chompunoot Wangboon

Suranaree University of Technology

\section{Patiwat Yasaka}

Rachamagala University

Chatanun Eamudomkarn

Khon Kaen University

Opal Pitaksakulrat

Khon Kaen University

Krisnakorn Tonkhamhak

Khon Kaen University

Arunee Paeyo

Khon Kaen University

Thomas Crellen

Oxford University: University of Oxford

Jiraporn Sithithaworn

Khon Kaen University 
Paiboon Sithithaworn ( $\sim$ paibsit@gmail.com )

Khon Kaen University

\section{Research Article}

Keywords: Mini Parasep SF stool concentrator kit, Kato-Katz, Formalin-ethyl acetate concentration technique, Diagnostic performance, Opisthorchiasis, helminthiasis, Opisthorchis viverrini

Posted Date: March 7th, 2022

DOI: https://doi.org/10.21203/rs.3.rs-1316646/v1

License: (c) (i) This work is licensed under a Creative Commons Attribution 4.0 International License. Read Full License 


\section{Abstract \\ Background}

Control and elimination of the liver fluke (Opisthorchis viverrini) is a primary preventive strategy against cholangiocarcinoma in Southeast Asia. A sensitive parasitological diagnostic method is required to facilitate such a surveillance and control program. In this study, we evaluated the performance of Mini Parasep SF stool concentrator kit (stool kit) in comparison with Kato-Katz (KK) and the quantitative formalin-ethyl acetate concentration technique (FECT) for detection of $O$. viverrini and coexisted parasitic infections.

\section{Methods}

A cross-sectional survey of parasitic infection of residents age $<15$ years old in a community in Kalasin province, northeast Thailand was conducted in 2018. Fecal samples were collected and screened by KK method and a subset of samples was examined by the stool kit and FECT methods. The results were analyzed for prevalence of parasitic infections and diagnostic performances of methods for qualitative and quantitative detection of helminthiasis.

\section{Results}

The initial survey of parasitic infection determined by the KK method $(n=567)$ showed the prevalence of O. viverrini was $32.63 \%$, followed by Taenia $2.65 \%$, echinostomes $1.76 \%$, hookworms $1.41 \%$, Trichuris trichiura $0.53 \%$, and Strongyloides stercoralis $0.53 \%$. Within a subset of samples tested with multiple diagnostics $(n=150)$, the detection rates of 0 . viverrini by the stool kit, FECT, and KK methods were $27.3 \%$, $30.7 \%$, and $28.7 \%$, respectively. The diagnostic sensitivity for opisthorchiasis were similar for FECT $(75.4 \%)$ and KK $(70.5 \%)$ and was lower for the stool kit $(67.2 \%, p<0.05)$. For other parasitic infections, FECT and stool kit performed better than KK methods particularly in detecting minute intestinal fluke (MIF), S. stercoralis, and coinfections. The intensity of $O$. viverrini infection (fecal egg counts) by the stool kit had significant positive correlations with KK as well as FECT $(p<0.05)$.

\section{Conclusions}

As the stool kit is simple to use and showed a comparable performance to FECT, it may serve as an alternative method of fecal examination for screening of helminthiasis including opisthorchiasis.

\section{Background}

Opisthorchis viverrini infection is a major public health problem in the Mekong River Basin region of Southeast Asia, especially in Thailand, the Lao People's Democratic Republic (Lao PDR), Cambodia, and 
Vietnam [1, 2]. The clinical sequela of chronic opisthorchiasis is hepatobiliary pathology; particularly intrahepatic cholangiocarcinoma (CCA) [3, 4]. Consequently, O. viverrini has been classified as a Group I biological carcinogen by the International Agency on Research in Cancer [5]. As 0 . viverrini infection has a fundamental role in the induction of CCA and the resulting fatalities, a comprehensive strategy for prevention, control, and elimination of $O$. viverrini is a prerequisite step toward reduction of the incidence of CCA [6].

After decades of chemotherapeutic control and improvement of public health efforts to control the parasitic diseases in Thailand, the epidemiology of $O$. viverrini infection changed dramatically [7]. Currently, O. viverrini infections are light (egg/gm feces $<50$ ) and spread across extensive geographical regions, especially in North and Northeastern Thailand, where control programs have been implemented for decades $[2,8,9]$. Screening of individuals with opisthorchiasis for surveillance and control in endemic areas has traditionally relied on conventional parasitological methods i.e., Kato-thick smear [10], formalin ethyl-acetate concentration technique (FECT), Kato-Katz (KK) method, and simple smear method [11]. These methods are known to have several drawbacks, including limited analytical sensitivity i.e., low intensity infections can go undetected and may require repeated fecal examination over several days. In addition, the KK method has been shown to have limited analytical specificity, with $O$. viverrini eggs often confused with the eggs from minute intestinal fluke (MIF) infection and accurate distinction requires experienced microscopists $[12,13,14,15]$. A previous study suggested that FECT is superior to the KK method for screening of $O$. viverrini, but FECT is logistically complicated i.e., samples require centrifugation and has practical disadvantages compared with the KK method [16]. To ease the complications of performing FECT, stool concentrator kits such as Mini Parasep ${ }^{\circledR}$ Kit have been developed to minimize specimen handling with a disposable enclosed system [17]. In addition, after specimen preparation, the samples can be kept for later examination which eases time demands when examining large numbers of samples. Initial studies using the stool kit have suggested that the method has comparable or better diagnostic performance than the direct simple smear, however, it was reported to be less sensitive than the FECT and KK method for diagnosis of 0 . viverrini $[16,18]$. We previously compared the stool kit against FECT and found that the stool kit method was less sensitive in the detection of $O$. viverrini but had a comparable performance for other co-endemic helminth infections [19]. As the results from these preliminary studies have been conflicting and based on small sample sizes, more data are needed to further evaluate the performance of the stool kit for detecting $O$. viverrini as well as other helminth infections in different transmission settings.

The objective of this study is to perform a cross-sectional survey of parasitic helminth infection in an endemic area of opisthorchiasis in northeast Thailand and evaluate the diagnostic performances of the fecal examination methods including the Mini Parasep SF stool concentrator kit, KK, and FECT for the diagnosis of $O$. viverrini and other parasitic infections. Relationships of quantitative data i.e., fecal egg counts of $O$. viverrini estimated by each method were also examined. The results from this study are informative on the current status of parasitic infection in this region and provide additional results on the analytical performance of the commercial stool kit in an endemic community in northeast Thailand and 
its feasibility for applications in larger field studies for surveillance and control of opisthorchiasis in Thailand as well as other $O$. viverrini endemic Southeast Asian countries.

\section{Methods}

\section{Study participants and sample collection}

A cross-sectional survey was carried out from March to July 2018 in an endemic area for infection with $O$. viverrini and other helminth parasites in Na Mon district, Kalasin province, northeast Thailand (Fig. 1). Residents aged 15 years or older, both men and women, were recruited in this study. After written informed consent was obtained, the participants were registered for demographic information. Clean plastic containers were labeled with participants' names and identifying numbers and were distributed to the villagers to collect the fecal samples. Approximately $10 \mathrm{~g}$ of fecal sample was obtained from each participant and the samples were kept in a chilled insulated box and transported from the study site to the laboratory at the Khon Kaen University. Each fecal sample was mixed and divided into 3 parts and processed for examinations by Mini Parasep SF stool concentrator kit method, FECT, and KK methods. The study population mostly comprised farmers working in paddy fields.

\section{Methods and procedures for fecal examination}

\section{Stool kit method}

The procedure for the Mini Parasep SF fecal parasite concentrator kit method (Diasys Europe, Berkshire, UK) was performed according to the manufacturer's instructions. In short, a fecal sample ( 0.5 g) was introduced into a tube containing $3.3 \mathrm{ml}$ of $10 \%$ formalin solution, and one drop of Triton $\mathrm{x}-100$ then was added. The sample was vortexed to emulsify the mixture. The tube was inverted and centrifuged at $500 \mathrm{~g}$ for 2 minutes and then the top layers of the supernatant were decanted. The pellet was resuspended in 1 $\mathrm{ml}$ of $10 \%$ formalin solution and two drops, mixed with Lugol's iodine solution, were examined under a light microscope (100x - 400x magnification). Enumerations of egg per gram feces (EPG) were similar to the procedure in modified FECT (see below). Discrimination between eggs of $O$. viverrini and minute intestinal fluke (MIF) i.e., lecithodendrid eggs, was based on morphological characteristics as previously described $[13,20]$.

\section{Formalin-ethyl acetate concentration technique (FECT)}

Fecal examination using a modified FECT diagnostic method was conducted as reported previously [21, 22]. Briefly, fecal samples were homogenized, and two grams of fresh feces were weighed and diluted in $7 \mathrm{~mL}$ of $10 \%$ formalin solution and strained through two layers of gauze into the $15 \mathrm{~mL}$ centrifuge tube. The strained suspension was centrifuged at 2,500 rpm for $5 \mathrm{~min}$, room temperature, and the supernatant was poured out. Thereafter, $7 \mathrm{~mL}$ of $0.85 \%$ saline were added to the tube and then vigorously mixed with $3 \mathrm{ml}$ of ethyl acetate for 30-60 seconds to aid in the extraction of fat from the feces. The suspension was then centrifuged at 2,500 rpm for $5 \mathrm{~min}$ at room temperature and the pellet was resuspended and fixed 
with $1 \mathrm{~mL}$ of $10 \%$ formalin. The final fecal suspension was examined two times (two drops) per sample by the same microscopist using a compound microscope at 100x and 400x magnifications with the results combined and multiplied by the number of drops in the suspension and divided by the mass of stool in grams to calculate the number of eggs per gram of feces (EPG). Morphological differentiation between eggs of $O$. viverrini and minute intestinal fluke was done as previously described by Kaewkes [13].

\section{Kato-Katz method}

After thorough homogenization of the samples, approximately one gram of feces was pressed through a mesh screen to remove large particles. Then a portion of the sieved sample was transferred to the standard template holding on a slide. After filling the hole, the template was removed, and the remaining sample was covered with a piece of cellophane pre-soaked (24 hours) in the $3 \%$ malachite green-glycerol solution. The slide was allowed to clear for $30 \mathrm{~min}$ before examination under a microscope. The number of eggs was counted and recorded for each helminth species separately and multiplied by 23 to calculate the EPG $[23,24]$.

\section{Ethics statement}

This human subject protocol was approved by the Ethics Committee of Khon Kaen University, Khon Kaen Thailand (reference number HE601370). Permission for fieldwork was obtained from the Ministry of Public Health (MOPH) and the provincial, district, and sub-district health officers. Written informed consents were obtained from all participating subjects. At the end of the project, infected individuals were treated with appropriate anthelmintic drugs.

\section{Statistical analysis}

Data were recorded in case report forms, entered into an Excel worksheet (Microsoft), and analyzed using SPSS 26 (IBM, Chicago, IL, USA). Data from individuals with matched fecal samples by the three diagnostic tests were considered for analysis. Positive fecal examination results referred to the presence of parasite eggs or larva in the fecal specimen examined by any one of three methods: stool kit, FECT, or KK. In the analysis for diagnostic performance of each method, the gold standard (100\% specificity and sensitivity) was defined as a combined result from the other two methods. Diagnostic accuracy in terms of clinical sensitivity, clinical specificity, positive predictive values (PPV), negative predictive values (NPV), and $95 \%$ confidence intervals $(95 \% \mathrm{Cl})$ for total parasites and $O$. viverrini separately were determined using MedCalc (Med Calc Software, Ostend, Belgium). The agreement in the status of $O$. viverrini and other helminth infections between two different methods was evaluated using Cohen's kappa coefficient. Cohen's kappa values $<0$ were interpreted as indicating no agreement and 0 0.20 as slight, $0.21-0.40$ as fair, $0.41-0.60$ as moderate, $0.61-0.80$ as substantial, and $0.81-1$ as almost perfect agreement on the status of $O$. viverrini and other helminth infection of the study participants between different methods [25]. McNemar's chi-squared test was used to compare the prevalence of $O$. viverrini and other parasitic infections between diagnostic methods. 
The intensity of $O$. viverrini infection determined as eggs per gram of feces (EPG) was categorized into three groups as 1-50, 51-100, and >100 [26]. Kendall's tau-b correlation test was used to determine the correlation of intensity (EPG) between two different methods. A statistically significant level was set at a $P$-value of $<0.05$.

\section{Result}

\section{Prevalence of parasite infection}

From the survey of parasitic infection determined by KK ( $n=567), 185$ individuals were positive for $O$. viverrini infection giving a prevalence of $32.63 \%$. In addition, the observed prevalence for other parasitic helminths were $2.65 \%$ for Taenia spp., $1.76 \%$ for echinostomes, $1.41 \%$ for hookworms, $0.53 \%$ for Trichuris trichiura, and $0.53 \%$ for Strongyloides stercoralis. As shown in Fig. 2A, the prevalence profiles of 0 . viverrini increased slowly and peaked at an age $>60$ years. The prevalence of $O$. viverrini in males ( 96 of $241,39.75 \%$,) was higher than those in females (90 of $328,27.44 \%)\left(c^{2}=9.53, P<0.01\right)$. The age-intensity profiles measured by KK were highest at age $>60$ years and the intensity of $O$. viverrini infection in males was higher than those in females $(P<0.001)$ (Fig. 2B).

One hundred and fifty participants provided complete and adequate specimens for fecal analyses were recruited for further study (Fig. 3). The age range of the participants was 17 to 80 years old and 60 were males $(40.0 \%)$ and 90 were females $(60.0 \%)$. The remaining 417 individuals were excluded from the final analysis as they submitted an inadequate amount of stool. There was no significant difference for sex $(P>0.05)$ and age categories $(P>0.05)$ between the recruited and the excluded participants.

\section{Qualitative comparisons between diagnostic methods}

Fecal examination for 0 . viverrini by the stool kit, FECT, and KK methods gave comparable positive detection rates were $30.7 \%$ for $\mathrm{FECT}, \mathbf{2 8 . 7 \%}$ for $\mathrm{KK}$, and $27.3 \%$ for stool kit with no statistically significant difference between methods (Table 1). FECT and stool kit detected more positive cases with parasites such as Strongyloides stercoralis and MIF than the KK method (McNemar's chi-square test, $P<0.001$ ). For parasites other than $O$. viverrini, FECT and stool kit yielded comparable positive rates and both methods gave significantly higher positive rates than the KK method $(P<0.001)$. As for the detection of all parasite species combined, positive detection rates by FECT and stool kit were comparable but both methods were significantly greater than for the KK method (McNemar's chi-square test, $P<0.05$ ).

As shown in Table 2, the stool kit and FECT had significantly higher sensitivity (72.7\% and $74.2 \%)$ for detecting all parasite species than that of the KK method (McNemar's chi-square test, $P<0.05$ ). In the case of $O$. viverrini, there was no significant difference in the sensitivity and specificity of the stool kit and the other methods. The sensitivity of stool kit, FECT, and KK method was $67.3 \%, 75.5 \%$, and $66.0 \%$, respectively. FECT had the highest NPV (88.5\%) followed by stool kit (84.4\%) and KK (83.2\%). For detection of $O$. viverrini, there were substantial agreements between stool kit and FECT (Kappa=0.693), between stool kit and KK (Kappa=0.669), and between FECT and KK (Kappa=0.665). The test for 
agreement for all parasite detection revealed a significant correlation with a substantial agreement between stool kit and FECT (Kappa=0.626), between FECT and KK (Kappa=0.612), and moderate agreement between stool kit and KK (Kappa=0.567) (Table 3).

When the sample subjects were classified into intensity classes of $O$. viverrini infection according to EPG determined by FECT, both stool kit and KK methods yielded comparable detection rates in all intensity classes (Table 4). Regarding the multiple parasite species infections, 78 participants $(52.0 \%)$ were found infected with at least one helminth (Table 5).

Comparisons between the stool kit, FECT, and KK showed significant differences in the numbers of parasites recovered $(P<0.05)$. All three methods detected a comparable positive rate of single-species infection. Detailed analysis in the case of $O$. viverrini and MIF detection revealed that the KK method misclassified three cases of MIF eggs as $O$. viverrini, whereas mixed $O$. viverrini and MIF (24 of 150, $16.0 \%$ ) were detected by both stool kit and FECT. For the detection of mixed 0 . viverrini and MIF, 12 positive cases $(50.0 \%)$ were reported by stool kit and 16 positive cases $(66.7 \%)$ were detected by FECT. For MIF, 5 samples were detected as positive by the stool kit method and 6 samples by FECT.

\section{Quantitative comparisons}

The intensity of infection expressed as EPG of feces by stool kit and KK methods were positively correlated (Fig. 4). There were $11 / 43$ cases (25.6\%) with zero EPG by the stool kit and a range of 23-1,173 EPG enumerated by the KK method. Conversely, there were $9 / 41$ cases $(21.9 \%)$ of zero EPG by KK method, and a range of 14-36 EPG was retrieved by stool kit. The fecal egg counts by KK and FECT showed a significant positive correlation $(p<0.001)$ and there were $12 / 46$ and $9 / 43$ cases undetectable by $\mathrm{KK}$ and FECT, respectively. In the case of the FECT and stool kit method, a similar correlation was seen and there were $7 / 41$ and 12/46 cases undetected by FECT and stool kit, respectively.

\section{Discussion}

Based on the sample population in an endemic community in northeast Thailand, screening of parasitic infection by fecal examination methods i.e., FECT, stool kit, and KK method showed comparable accuracy for qualitative and quantitative diagnosis of $O$. viverrini. For diagnosis of all parasitic helminth infections, the FECT, and stool kit methods performed better than the KK method in discovering more species of coendemic parasitic infection; particularly MIF and $S$. stercoralis. The observation that the stool kit performs equally well as FECT for population screening of $O$. viverrini in addition to other helminth infections suggests its potential application in field operations for surveillance and monitoring effects of mass treatment for helminthiases including opisthorchiasis.

Analyses of the diagnostic performance of fecal examination methods revealed that for $O$. viverrini, all three methods had similar sensitivity, specificity, and predictive values. It is known that the KK method does not distinguish MIF from $O$. viverrini $[12,27,28]$, in this study, FECT and stool kit discovered 11.3$14.7 \%$ MIF compared to none by KK method $(n=150)$. Although adult worm expulsion and/or molecular 
confirmation is required for definite identification of MIF in question $[13,14,28,29]$, previous records in this part of northeast Thailand showed that $O$. viverrini is predominated and the prevalence of MIF was low [21, 30, 31]. By contrast, MIF specifically Haplorchis spp. were more abundant than 0 . viverrini in the central part of Lao PDR [32]. In northern Thailand, using molecular diagnostics the occurrence of MIF was recently found to be 3.8-fold higher than 0 . viverrini [33].

For diagnosis of all helminth infections combined, FECT and stool kit showed higher sensitivity than the KK method. The underlying reason is not only explained by the fact that the KK method could not differentiate between MIF and $O$. viverrini but also the KK method did not detect $S$. stercoralis larva in the fecal specimen. In case of echinostome and Taenia spp. eggs which are relatively large, the KK method gave a comparable detection rate to FECT and stool kit which was a similar finding to previous studies $[15,19,34,35]$. Diagnostic agreement between methods by Cohen's kappa tests showed that the kappa value ranged from moderate to substantial.

Since epidemiological surveys of parasitic helminth in Southeast Asia, including Thailand, frequently report coinfections with multiple species $[12,29,30,36]$, diagnostic methods which can reliably classify helminth eggs are preferred. While FECT, stool kit, and KK method were equally efficient in detecting eggs of $O$. viverrini, for multiple infections FECT and stool kit were better than KK method. The explanation for these discrepancies may depend on several factors. The first is related to the greater amount of fecal sample employed for the FECT $(2 \mathrm{~g})$ and stool kit $(0.5 \mathrm{~g})$ method compared with the KK method (40 $\mathrm{mg})$ [19]. Secondly, the processing steps in FECT and stool kit which filter out fecal debris as well as fat from fecal specimens contribute to improved egg isolation, hence increasing the chances of detecting parasite eggs or larvae. Thirdly, the protocol for fecal examination by FECT and stool kit was 2 replicates while it was one replicate for KK. If more replicates were examined in the $\mathrm{KK}$ method, the diagnostic performance may be improved $[35,37,38]$.

Quantitative analysis of fecal egg counts of $O$. viverrini indicated that FECT, stool kit, and KK method were similar regardless of the intensity of infection. Given the current trend of widespread low intensity, stool kit and KK method were equally sensitive to discover $O$. viverrini infected cases. In Korea and China, many studies have reported that the $\mathrm{KK}$ method was more suitable for $C$. sinensis than the formalin-ether technique and direct smear $[39,40]$. In Southeast Asia where $O$. viverrini is endemic both FCET and KK method with repeated stool examination is recommended $[35,37,38]$.

The KK method has been used for screening and control programs in Thailand for decades [7]. However, in current epidemiological settings in Thailand where light infection of $O$. viverrini is more common, the prevalence of $O$. viverrini, as well as coexisting infections measured by the $\mathrm{KK}$ method, is liable to underreporting [40]. While FECT requires specific laboratory equipment and is not practical in resourcepoor settings, the stool kit was designed to be an all-in-one tube with a fixative and built-in filtration apparatus and thus remove fecal debris similar to the procedure for FECT. In addition, it has an advantage over the $\mathrm{KK}$ method due to the extended storage time after sample preparation, and the sample can be processed and kept from examination later. The findings in our study that the stool kit 
performed equally well to FECT and better than the KK method for parasite survey in northeast Thailand suggested that it may be a method of choice for field application. However, a previous study showed that stool kit had lower sensitivity than the FECT and KK method and is not recommended for low-intensity infection [16]. To ascertain this discrepancy, it is needed to further apply the stool kit in endemic areas of opisthorchiasis with varying levels of transmission. An example of this approach has been demonstrated by comparing urine assay and FECT in areas with varying transmission levels of opisthorchiasis and the data suggested that urine assay was a robust and reliable method for population screening and diagnosis of opisthorchiasis [26].

This study was confined to a single locality and with a limited sample size; therefore further applications of the stool kit in multiple localities with varying transmission levels are required for a more comprehensive evaluation of its performance. The second limitation was the use of a single fecal examination in this study and it is known that increasing numbers of replicate examinations in each method may affect diagnostic performance. Thirdly, the separation of $O$. viverrini and MIF were based on morphological criteria so an independent technique such as molecular or adult worm expulsion is required for definite identification and taxonomic confirmation [11]. Evaluation of cost-effectiveness between methods which is essential for mass screening has not been addressed and deserves a separate, detailed study.

\section{Conclusion}

The results from this study demonstrate that the stool kit, FECT and KK methods had comparable diagnostic sensitivity for $O$. viverrini. The $\mathrm{KK}$ method had lower sensitivity for detection of overall parasitic infections compared with FECT and stool kit. The stool kit and FECT performed better for detection of MIF, S. stercoralis, and multiple infections than KK method. In terms of quantitative diagnosis measured of $O$. viverrini infection, fecal egg counts of the stool kit had a significant positive correlation with those by KK as well as by FECT. Overall, the stool kit has the potential for being a tool for screening of $O$. viverrini and coexisting parasitic infection. The utility of the stool kit to assess outcomes following anthelmintic treatment remains to be investigated.

\section{Abbreviations}

CCA: Cholangiocarcinoma; KK: Kato-Katz; stool kit: Mini Parasep SF kit; FECT: Formalin-ethyl acetate concentration technique; MIF: Minute intestinal fluke; EPG: Egg per gram feces; MOPH: Ministry of Public Health; PPV: Positive predictive values; NPV: Negative predictive values; $95 \% \mathrm{Cl}$ : $95 \%$ confidence intervals.

\section{Declarations}

Acknowledgments 
We thank the participants in Na Mon district and the Provincial Health Departments of Kalasin Province for their support and collaboration of the fieldwork.

\section{Authors' contributions}

KYK and PS conceived the study. PS, JS, NK and WL contributed to the conception and design of the study. KYK, PS and JS designed the study protocol. KYK, SS, C Worasith, CH, C Wangboon, PY, CE, OP, KT and AP carried out the field work for specimen collections and sample analysis. KYK, PS and TC carried out the analysis and interpretation of data. KYK and PS drafted the manuscript. KYK and PY designed the graphic results. PS and TC critically revised the manuscript for intellectual content. All authors read and approved the final manuscript. PS and NK are guarantors of the paper.

\section{Funding}

This work described here was supported by the Cholangiocarcinoma Research Institute, Khon Kaen University, and Fluke-Free Thailand, National Research Council of Thailand. TC is supported by a Sir Henry Wellcome Postdoctoral Fellowship. This research was funded in whole, or in part, by the Wellcome Trust (Grant number 215919/Z/19/Z). For the purpose of open access, the author has applied a CC BY public copyright licence to any Author Accepted Manuscript version arising from this submission.

\section{Availability of data and materials}

The datasets generated and/or analyzed during the current study are available from the corresponding author on reasonable request.

\section{Ethics approval and consent to participate}

The human experimental protocol was approved by the Khon Kaen University Ethics Committee (reference number HE601370). Only subjects who provided written informed consent were recruited. Treatment with a single oral dose of PZQ (40 mg/kg body weight) was given to subjects who were diagnosed as $O$. viverrini- positive by the FECT and/or urine antigen detection assay. Subjects who were found positive for other parasitic infections were given appropriate anthelmintic drugs.

\section{Consent for publication}

Not applicable.

\section{Competing interests}

The authors declare that they have no competing interests.

\section{Author details}


${ }^{1}$ Faculty of Public Health, Kasetsart University Chalermphrakiat Sakon Nakhon Province Campus, Sakon Nakhon, Thailand. ${ }^{2}$ Cholangiocarcinoma Research Institute, Khon Kaen University, Khon Kaen, Thailand. ${ }^{3}$ The Office of Disease Prevention and Control 7 Khon Kaen, Department of Disease Control, Ministry of Public Health, Thailand. ${ }^{4}$ Department of Surgery, Faculty of Medicine, Khon Kaen University, Khon Kaen,

Thailand. ${ }^{5}$ School of Pre clinic, Institute of Science, Suranaree University of Technology, Nakhon Ratchasima, Thailand. ${ }^{6}$ Faculty of Management Technology, Rajamangala University of Technology Isan, Surin Campus, Surin, Thailand. ${ }^{7}$ Department of Parasitology, Faculty of Medicine, Khon Kaen University, Khon Kaen, Thailand. ${ }^{8}$ Big Data Institute, Nuffield Department of Medicine, University of Oxford, Oxford, OX3 7LF, United Kingdom. ${ }^{9}$ Faculty of Associated Medical Sciences, Khon Kaen University, Khon Kaen, Thailand.

\section{References}

1. Andrews RH, Sithithaworn P, Petney TN. Opisthorchis viverrini: an underestimated parasite in world health. Trends Parasitol. 2008;24 11:497-501.

2. Sithithaworn $P$, Andrews RH, Nguyen VD, Wongsaroj T, Sinuon M, Odermatt $P$, et al. The current status of opisthorchiasis and clonorchiasis in the Mekong Basin. Parasitol Int. 2012;61(1):10-6.

3. Chamadol N, Khuntikeo N, Thinkhamrop B, Thinkhamrop K, Suwannatrai AT, Kelly M, et al. Association between periductal fibrosis and bile duct dilatation among a population at high risk of cholangiocarcinoma: a cross-sectional study of cholangiocarcinoma screening in Northeast Thailand. BMJ Open. 2019;9:3:e023217.

4. Khuntikeo N, Thinkhamrop B, Bundhamcharoen K, Andrews RH, Grundy-Warr C, Yongvanit P, et al. The socioeconomic burden of cholangiocarcinoma associated with Opisthorchis viverrini sensu lato infection in Northeast Thailand: A Preliminary Analysis. Adv Parasitol. 2018;102:141-63.

5. IARC. A Review of Human Carcinogens. Monographs on the Evaluation of Carcinogenic Risks to Humans: World Health Organization International Agency Research On Cancer 2011:100.

6. Crellen T, Sithithaworn P, Pitaksakulrat O, Khuntikeo N, Medley GF, Hollingsworth TD. Towards evidence-based control of Opisthorchis viverrini. Trends Parasitol. 2021;37 5:370-80.

7. Jongsuksuntigul P, Imsomboon T. Epidemiology of opisthorchiasis and national control program in Thailand. Southeast Asian J Trop Med Public Health. 1998;29(2):327-32.

8. Jongsuksuntigul P, Imsomboon T. The impact of a decade long opisthorchiasis control program in northeastern Thailand. Southeast Asian J Trop Med Public Health. 1997;28 3:551-7.

9. Prakobwong S, Suwannatrai K. Reduction of reinfection rates with Opisthorchis viverrini through a three-year management program in endemic areas of Northeastern Thailand. Korean J Parasitol. 2020;58 5:527-35.

10. Jongsuksuntigul P, Imsomboon T. Opisthorchiasis control in Thailand. Acta Trop. 2003;88 3:229-32.

11. Saijuntha W, Duenngai K, Tangkawattana S, Petney TN, Andrews RH, Sithithaworn P. Recent advances in the diagnosis and detection of Opisthorchis viverrini sensu lato in human and 
intermediate hosts for use in control and elimination programs. Adv Parasitol. 2018;101:177-214.

12. Chai JY, Darwin Murrell K, Lymbery AJ. Fish-borne parasitic zoonoses: status and issues. Int J Parasitol. 2005;35(11-12):1233-54.

13. Kaewkes S. Taxonomy and biology of liver flukes. Acta Trop. 2003;88 3:177-86.

14. Lovis L, Mak TK, Phongluxa K, Soukhathammavong P, Sayasone S, Akkhavong K, et al. PCR diagnosis of Opisthorchis viverrini and Haplorchis taichui infections in a Lao community in an area of endemicity and comparison of diagnostic methods for parasitological field surveys. J Clin Microbiol. 2009;47 5:1517-23.

15. Sayasone S, Vonghajack Y, Vanmany M, Rasphone O, Tesana S, Utzinger J, et al. Diversity of human intestinal helminthiasis in Lao PDR. Trans R Soc Trop Med Hyg. 2009;103(3):247-54.

16. Charoensuk L, Subrungruang I, Mungthin M, Pinlaor S, Suwannahitatorn P. Comparison of stool examination techniques to detect Opisthorchis viverrini in low intensity infection. Acta Trop. 2019;191:13-6.

17. Saez AC, Manser MM, Andrews N, Chiodini PL. Comparison between the Midi Parasep and Midi Parasep Solvent Free (SF) faecal parasite concentrators. J Clin Pathol. 2011;64 10:901-4.

18. Kaewpitoon SJ, Rujirakul R, Tongtawee T, Matrakul L, Panpimanmas S, Wakkuwattapong P, et al. Detection of the carcinogenic liver fluke Opisthorchis viverrini using a Mini Parasep SF faecal parasite concentrator. Asian Pac J Cancer Prev. 2016;17(1):373-6.

19. Laoprom N, Laithavewat L, Kopolrat K, Kiatsopit N, Kaewkes S, Chantalux S, et al. Evaluation of a commercial stool concentrator kit compared to direct smear and formalin-ethyl acetate concentration methods for diagnosis of parasitic infection with special reference to Opisthorchis viverrini sensu lato in Thailand. Southeast Asian J Trop Med Public Health. 2016;47 5:890-900.

20. Kaewkes S, Elkins DB, Sithithaworn P, Haswell-Elkins MR. Comparative studies on the morphology of the eggs of Opisthorchis viverrini and lecithodendriid trematodes. Southeast Asian J Trop Med Public Health. 1991;22(4):623-30.

21. Elkins DB, Sithithaworn P, Haswell-Elkins M, Kaewkes S, Awacharagan P, Wongratanacheewin S. Opisthorchis viverrini: relationships between egg counts, worms recovered and antibody levels within an endemic community in northeast Thailand. Parasitology. 1991;102 Pt 2:283-8.

22. Worasith C, Kamamia C, Yakovleva A, Duenngai K, Wangboon C, Sithithaworn J, et al. Advances in the diagnosis of human opisthorchiasis: development of Opisthorchis viverrini antigen detection in urine. PLoS Negl Trop Dis. 2015;9 10:e0004157.

23. Kato K, Miura M. Comparative examinations. Japanese J Parasitol. 1954;3:35.

24. Katz N, Chaves A, Pellegrino J. A simple device for quantitative stool thick-smear technique in Schistosomiasis mansoni. Rev Inst Med Trop Sao Paulo. 1972;14 6:397-400.

25. Landis JR, Koch GG. The measurement of observer agreement for categorical data. Biometrics. 1977;33(1):159-74. 
26. Worasith C, Wangboon C, Duenngai K, Kiatsopit N, Kopolrat K, Techasen A, et al. Comparing the performance of urine and copro-antigen detection in evaluating Opisthorchis viverrini infection in communities with different transmission levels in Northeast Thailand. PLoS Negl Trop Dis. 2019;13:2:e0007186.

27. Buathong S, Leelayoova S, Mungthin M, Ruang-Areerate T, Naaglor T, Suwannahitatorn P, et al. Molecular discrimination of Opisthorchis-like eggs from residents in a rural community of central Thailand. PLoS Negl Trop Dis. 2017;11:11:e0006030.

28. Lamaningao P, Kanda S, Laimanivong S, Shimono T, Darcy AW, Phyaluanglath A, et al. Development of a PCR assay for diagnosing trematode (Opisthorchis and Haplorchis) infections in human stools. Am J Trop Med Hyg. 2017;96(1):221-8.

29. Chai JY, Shin EH, Lee SH, Rim HJ. Foodborne intestinal flukes in Southeast Asia. Korean J Parasitol. 2009;47(Suppl):69-102.

30. Boonjaraspinyo S, Boonmars T, Kaewsamut B, Ekobol N, Laummaunwai P, Aukkanimart R, et al. A cross-sectional study on intestinal parasitic infections in rural communities, northeast Thailand. Korean J Parasitol. 2013;51 6:727-34.

31. Ramsay RJ, Sithithaworn P, Prociv P, Moorhouse DE, Methaphat C. Density-dependent fecundity of Opisthorchis viverrini in humans, based on faecal recovery of flukes. Trans R Soc Trop Med Hyg. 1989;83(2):241-2.

32. Chai JY, Yong TS, Eom KS, Min DY, Jeon HK, Kim TY, et al. Hyperendemicity of Haplorchis taichui infection among riparian people in Saravane and Champasak Province, Lao PDR. Korean J Parasitol. 2013;51 3:305-11.

33. Buathong S, Phaiphilai K, Ruang-Areerate T, Sitthichot N, Thita T, Mungthin M, et al. Genetic Differentiation of Opisthorchis-like eggs in northern Thailand using stool specimens under national strategic plan to control liver fluke infection and cholangiocarcinoma. Am J Trop Med Hyg. 2020;103 3:1118-24.

34. Funk AL, Boisson S, Clasen T, Ensink JH. Comparison of Kato-Katz, ethyl-acetate sedimentation, and Midi Parasep $(\mathrm{R})$ in the diagnosis of hookworm, Ascaris and Trichuris infections in the context of an evaluation of rural sanitation in India. Acta Trop. 2013;126 3:265-8.

35. Sayasone S, Utzinger J, Akkhavong K, Odermatt P. Repeated stool sampling and use of multiple techniques enhance the sensitivity of helminth diagnosis: a cross-sectional survey in southern Lao People's Democratic Republic. Acta Trop. 2015;141 Pt B:315-21.

36. Sayasone S, Utzinger J, Akkhavong K, Odermatt P. Multiparasitism and intensity of helminth infections in relation to symptoms and nutritional status among children: a cross-sectional study in southern Lao People's Democratic Republic. Acta Trop. 2015;141 Pt B:322-31.

37. Lamberton PH, Kabatereine NB, Oguttu DW, Fenwick A, Webster JP. Sensitivity and specificity of multiple Kato-Katz thick smears and a circulating cathodic antigen test for Schistosoma mansoni diagnosis pre- and post-repeated-praziquantel treatment. PLoS Negl Trop Dis. 2014;8:9:e3139. 
38. Qian MB, Zhuang SF, Zhu SQ, Deng XM, Li ZX, Zhou XN. Improving diagnostic performance of the Kato-Katz method for Clonorchis sinensis infection through multiple samples. Parasit Vectors. 2019;12 1:336.

39. Choi MH, Ge T, Yuan S, Hong ST. Correlation of egg counts of Clonorchis sinensis by three methods of fecal examination. Korean J Parasitol. 2005;43 3:115-7.

40. Hong ST, Choi MH, Kim CH, Chung BS, Ji Z. The Kato-Katz method is reliable for diagnosis of Clonorchis sinensis infection. Diagn Microbiol Infect Dis. 2003;47(1):345-7.

\section{Tables}

Table 1 Parasite detection rates determined by Mini Parasep SF kit (stool kit), formalin-ethyl acetate concentration technique (FECT), and Kato-Katz (KK) method and statistical comparisons between methods $(n=150)$. Data shown are the number of positive cases (percentage) and $p$-values from McNemar's chi-square test.

\begin{tabular}{|c|c|c|c|c|c|c|}
\hline \multirow[t]{2}{*}{ Parasites } & \multicolumn{3}{|c|}{ No. positive (\%) } & \multicolumn{3}{|l|}{$p$ values } \\
\hline & Stool Kit & FECT & KK & $\begin{array}{l}\text { Stool } \\
\text { Kit vs FECT }\end{array}$ & $\begin{array}{l}\text { Stool } \\
\text { Kit vs KK }\end{array}$ & $\begin{array}{l}\text { FECT vs } \\
\text { KK }\end{array}$ \\
\hline Opisthorchis viverrini & $41(27.3)$ & $46(30.7)$ & $43(28.7)$ & $>0.05$ & $>0.05$ & $>0.05$ \\
\hline \multicolumn{7}{|l|}{ Other parasites } \\
\hline $\begin{array}{l}\text { Minute intestinal } \\
\text { fluke }\end{array}$ & $17(11.3)$ & $22(14.7)$ & $0(0.0)$ & & & \\
\hline $\begin{array}{l}\text { Strongyloides } \\
\text { stercoralis }\end{array}$ & $16(10.7)$ & $12(8.0)$ & $0(0.0)$ & & & \\
\hline Echinostome eggs & $3(2.0)$ & $2(1.3)$ & $3(2.0)$ & & & \\
\hline Taenia sp. & $2(1.3)$ & $3(2.0)$ & $3(2.0)$ & & & \\
\hline Hookworm eggs & $3(2.0)$ & $0(0.0)$ & $0(0.0)$ & & & \\
\hline Ascaris lumbricoides & $0(0.0)$ & $1(0.7)$ & $0(0.0)$ & & & \\
\hline Trichuris trichiura & $0(0.0)$ & $1(0.7)$ & $0(0.0)$ & & & \\
\hline \multirow{2}{*}{$\begin{array}{l}\text { Combined other } \\
\text { parasites }\end{array}$} & $41(27.3)$ & $41(27.3)$ & $6(4.0)$ & $>0.05$ & $<0.001$ & $<0.001$ \\
\hline & $60(40.0)$ & $61(40.7)$ & $46(30.7)$ & $>0.05$ & $<0.05$ & $<0.05$ \\
\hline
\end{tabular}

Table 2 Diagnostic accuracy of the stool kit, formalin-ethyl acetate concentration technique (FECT), and Kato-Katz (KK) in the diagnosis of overall parasitic infection and Opisthorchis viverrini in fecal samples using combined result from the other two methods as a reference diagnosis. 


\begin{tabular}{|lllll|}
\hline Method & Sensitivity & Specificity & PPV & NPV $^{\mathrm{a}}$ \\
\hline Overall parasites & & & & \\
\hline Stool Kit & $72.7(60.4-83.0)$ & $85.7(76.4-92.4)$ & $80.0(69.9-87.3)$ & $80.0(72.8-85.7)$ \\
\hline FECT & $74.2(62.0-84.2)$ & $85.7(76.4-92.4)$ & $80.3(70.4-87.5)$ & $80.9(73.6-86.6)$ \\
\hline KK & $56.8(44.7-68.2)$ & $94.7(87.1-98.5)$ & $91.3(79.9-96.5)$ & $69.2(63.3-74.6)$ \\
\hline Opisthorchis viverrini & & & & \\
\hline Stool Kit & $67.3(52.9-79.8)$ & $93.9(87.2-97.7)$ & $85.4(72.4-92.8)$ & $84.4(78.5-88.9)$ \\
\hline FECT & $75.5(61.1-86.7)$ & $91.1(83.8-95.8)$ & $80.4(68.4-88.7)$ & $88.5(82.4-92.6)$ \\
\hline KK & $66.0(51.7-78.5)$ & $91.8(84.4-96.4)$ & $81.4(68.7-89.7)$ & $83.2(77.2-87.9)$ \\
\hline
\end{tabular}

a\% (95\% Cl), Positive Predictive Value (PPV), Negative Predictive Value (NPV)

Table 3 Diagnostic agreements between stool kit, FECT, and KK method for diagnosis of overall parasite and Opisthorchis viverrini infection. Data shown were Cohen's kappa statistics.

\begin{tabular}{|ccccc|}
\hline \multicolumn{1}{|c|}{ Variable } & Kappa & \multicolumn{2}{c|}{$95 \%$ Confidence Interval } & P-values \\
\cline { 3 - 5 } & & Lower & Upper & \\
Overall parasite & & & & \\
Stool Kit vs FECT & 0.626 & 0.499 & 0.753 & $<0.001$ \\
\hline Stool Kit vs KK & 0.567 & 0.432 & 0.701 & $<0.001$ \\
\hline FECT vs KK & 0.612 & 0.483 & 0.741 & $<0.001$ \\
\hline Opisthorchis viverrini & & & & \\
\hline Stool Kit vs FECT & 0.693 & 0.565 & 0.820 & $<0.001$ \\
\hline Stool Kit vs KK & 0.669 & 0.536 & 0.803 & $<0.001$ \\
\hline FECT vs KK & 0.665 & 0.533 & 0.796 & $<0.001$ \\
\hline
\end{tabular}

Table 4 Prevalence rates of Opisthorchis viverrini determined by the stool kit (Mini Parasep SF kit) and Kato-Katz (KK) in different infection intensity groups classified by fecal egg counts (EPG) by quantitative FECT. Comparisons between prevalence rates in intensity class by McNemar's chi-square tests showed no significant differences $(p>0.05)$. 


\begin{tabular}{|lllll|}
\hline The intensity of O. viverrini infection (EPG) & $\mathrm{N}$ & \multicolumn{2}{l}{ No. positive (\%) } & p values \\
\cline { 3 - 5 } & & Stool kit & KK & Stool kit vs KK \\
\hline 0 & 104 & $7(6.7)$ & $9(8.7)$ & 0.791 \\
\hline $1-50$ & 32 & $22(68.8)$ & $21(65.6)$ & 1.000 \\
\hline $51-100$ & 7 & $6(85.7)$ & $7(100.0)$ & 1.000 \\
\hline$>100$ & 7 & $6(85.7)$ & $6(85.7)$ & 1.000 \\
\hline Total & 150 & $41(27.3)$ & $43(28.7)$ & 0.824 \\
\hline
\end{tabular}

Table 5 Effect of multiple infections on the performance of Mini Parasep SF kit (stool kit), formalin-ethyl acetate concentration the technique (FECT) and Kato-Katz (KK) method.

\begin{tabular}{|cllllll|}
\hline \multirow{2}{*}{$\begin{array}{c}\text { Infection } \\
\text { category }\end{array}$} & \multicolumn{2}{l}{ No. positive (\%) } & \multicolumn{3}{l|}{ Comparisons $(p \text { values) })^{\text {a }}$} \\
\cline { 2 - 7 } & Stool Kit & FECT & KK & $\begin{array}{l}\text { Stool } \\
\text { Kit vs FECT }\end{array}$ & $\begin{array}{l}\text { Stool Kit vs } \\
\text { KK }\end{array}$ & $\begin{array}{l}\text { FECT vs } \\
\text { KK }\end{array}$ \\
\hline Single infection & $41(27.3)$ & $40(26.7)$ & $43(28.7)$ & 1.000 & 0.880 & 0.749 \\
\hline $\begin{array}{c}\text { Double } \\
\text { infection }\end{array}$ & $16(10.7)$ & $16(10.7)$ & $3(2.0)$ & 1.000 & 0.002 & 0.002 \\
$\begin{array}{c}\text { Multiple } \\
\text { infection }\end{array}$ & $3(2.0)$ & $5(3.3)$ & $0(0.0)$ & 0.688 & 0.250 & 0.063 \\
\hline
\end{tabular}

${ }^{a}$ McNemar chi-square test.

\section{Figures}

\section{Figure 1}

Map of the study area showing an overview of an endemic area for opisthorchiasis in Na Mon district, Kalasin province, northeast Thailand. 


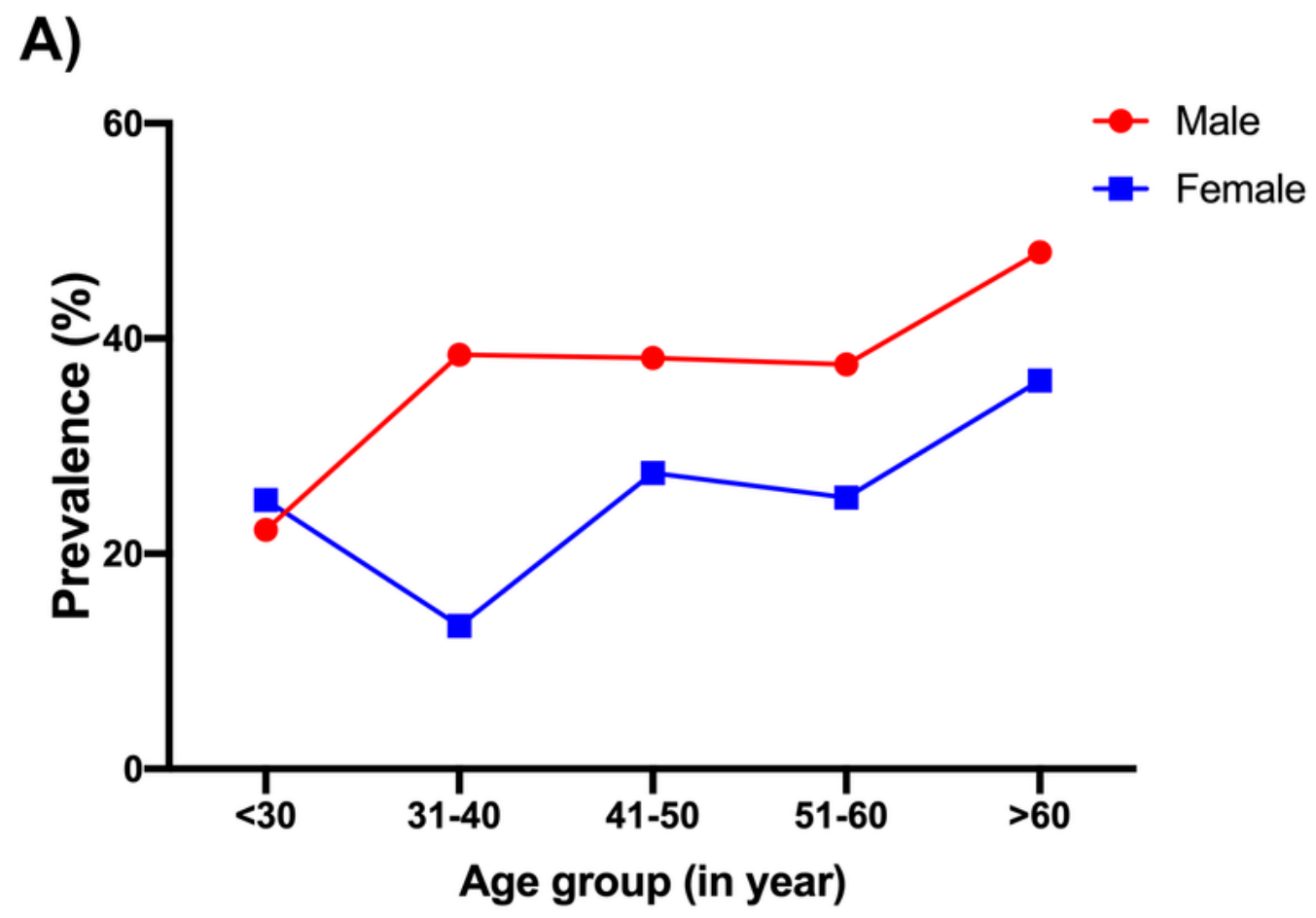

B)

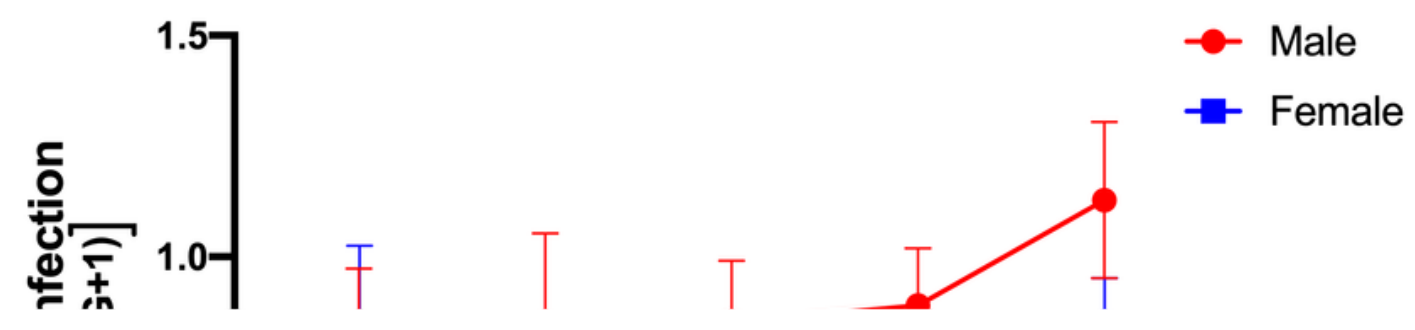

Figure 2

Age-sex prevalence (A) and age-sex intensity (B) profiles of 0 . viverrini determined by Kato Katz method $(n=567)$. 


\section{Figure 3}

Flow diagram of study participants and sample collection. Data shown for age are mean $\pm S D$, where $n$ is the sample size. 

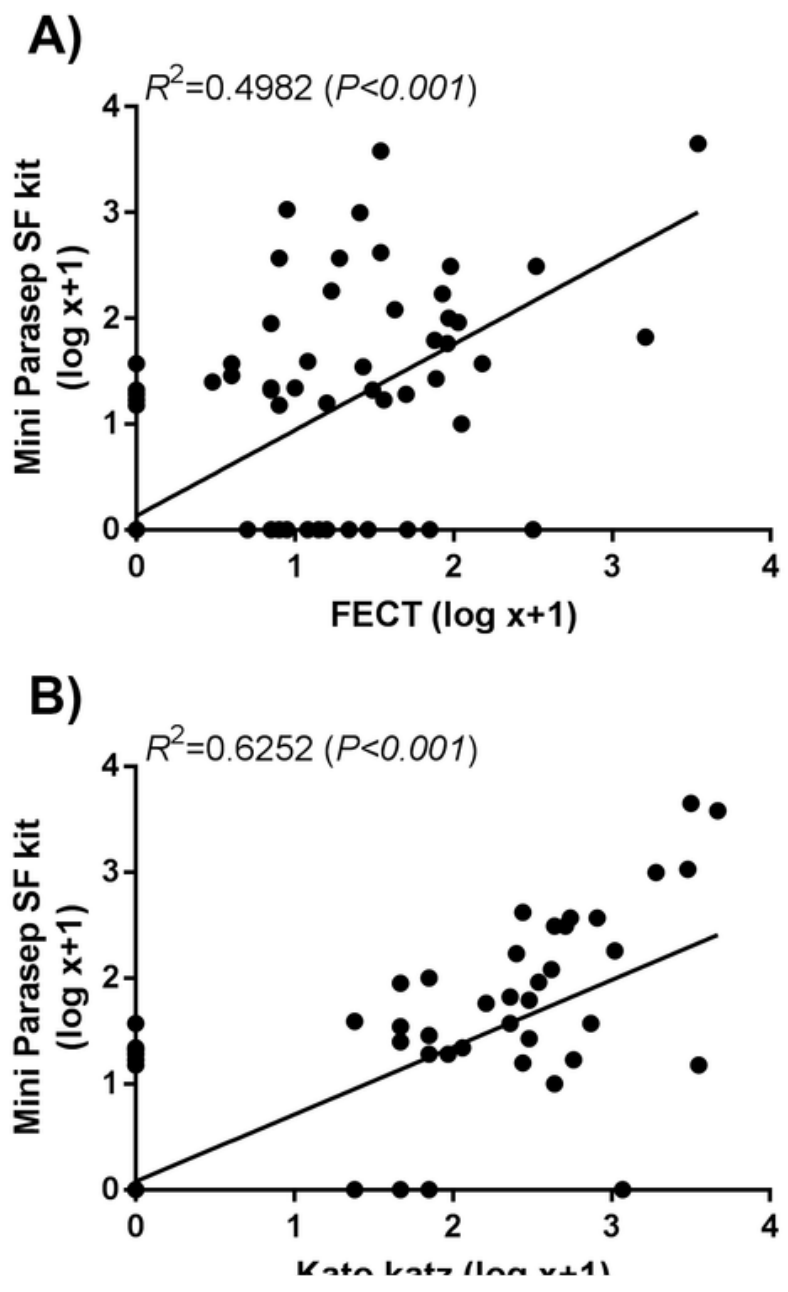

Figure 4

Relationship between eggs per gram of feces of $O$. viverrini measured by Mini Parasep SF kit (stool kit) and formalin-ethyl acetate concentration technique (FECT) (A), stool kit and Kato-Katz (KK) (B), and FECT and KK methods (C). The dots shown are observed data and a solid line represented a linear regression equation. $R^{2}$ is the correlation coefficient and $P$-value. 


\section{Supplementary Files}

This is a list of supplementary files associated with this preprint. Click to download.

- GraphAbst.tif 